\title{
On the Mode of Transmission of Mycobacterium leprae
}

\author{
D. L. LEIKER \\ Royal Tropical Institute, Amsterdam, The Netherlands
}

\begin{abstract}
The finding that in patients with lepromatous leprosy much larger numbers of bacilli are released from the nasal mucosa and from the milk ducts of lactating mothers as compared with those present on the surface of the skin, suggests the possibility of transmission by droplet infection and by breast feeding and the consequent possibility that the primary lesions are located in the respiratory and gastro-intestinal tract and that the skin lesions are secondary lesions. Clinical and epidemiological evidence against this hypothesis is presented, and it is concluded that droplet infection via the respiratory tract is not a common mode of transmission, and the present trend of abandoning segregation and other restrictive measures against leprosy patients should not be reversed.
\end{abstract}

\section{Introduction}

The concept that leprosy is a feebly contagious disease, mainly spread by prolonged, intimate direct skin contact between highly bacilliferous patients and susceptible healthy subjects, can no longer be held to be valid. It has been difficult for some time to maintain the concept of feeble contagiousness in view of the epidemics of leprosy recorded from various countries (Wade and Ledowsky, 1952; Davey, 1957; Leiker, 1971) with 10-30\% of the population developing symptoms of leprosy within a few decades of the introduction of the disease into the community. Recently, Godal et al. (1974) produced immunological evidence that sub-clinical infections are common. In people who had lived for some years in a country with endemic leprosy, a significantly higher percentage of positive lymphocyte transformation tests, after incubation with Mycobacterium leprae, was found as compared with recent immigrants from non-endemic areas. Apparently the rate of transmission of $M$. leprae is significantly higher than is indicated by prevalence surveys.

The concept of prolonged, intimate contact is also seriously challenged by the actual findings in epidemics of leprosy. Frequently, in whole-population surveys of villages with a very high leprosy prevalence, only a single one, or at most very few highly bacilliferous patients are found, and not rarely none are encountered, even though cases of leprosy are to be found in nearly all families in the community. It is inconceivable that so many patients in unrelated families can have had prolonged, intimate contact with the few highly bacilliferous patients 
present, because the intensity of contact in such communities is largely governed by family relationships. It is much more likely that in a high proportion of the patients leprosy was transmitted after short, superficial, direct contact with the few highly bacilliferous patients or with other, only moderately strong bacteriologically positive patients, or that indirect contact plays a major role, e.g. transmission by flies (Geater, 1975).

In the Netherlands, more than $50 \%$ of the 1000 recorded patients-immigrants from endemic countries were unable to recall any prolonged, intimate contact with highly bacilliferous patients, and in the great majority there is no reason to doubt their statements. Some Dutch patients have contracted leprosy after a brief sojourn in an endemic country, without being able to recall any contact at all with leprosy patients. One Dutchman, who had never left the country and who had no known contact with leprosy patients, developed lepromatous leprosy (Beek, 1961).

Recently, the concept of skin contact as the main mode of transmission has been challenged as well. Pedley (1970) has shown that the number of bacilli present on the surface of the skin of highly bacilliferous patients is relatively low as compared with the large numbers of bacilli released by the nasal mucosa. Large numbers of bacilli are also present in the secretory cells of the milk glands of lactating lepromatous mothers (Pedley, 1968). The theoretical possibility of spreading $M$. leprae by droplet infection is supported by experiments in mice with aerosols containing $M$. leprae (Rees et al., 1976). These findings suggest that $M$. leprae might be spread by droplet infection and by breast feeding, and that the primary lesion of leprosy is located in the respiratory or in the gastro-intestinal tract and not in the skin. Rees and Meade (1974) have stated that the attack rate of leprosy and of tuberculosis are similar and that this finding, though not proof of identical modes of spread, is consistent with this possibility.

First, the possibility of transmission via the skin will be discussed.

\section{Transmission via the Skin}

It has been assumed that $M$. leprae is unable to pass through unbroken skin. It should, however, be emphasized that the skin is seldom unbroken, minor scratches and wounds usually being present.

In lepromatous leprosy large numbers of bacilli are usually present in the upper part of the corium. Though separated by a subepidermal zone which is usually free of bacilli, a minor wound or scratch may bring bacilli to the surface of the skin. Large numbers of bacilli may also be present in superficial lesions in reactive lepromatous patients, especially if the reaction is vesicular, bullous or ulcerating. Such lesions are most vulnerable and rupture of the bullae results in the temporary presence of large numbers of bacilli on the surface of the skin. But also in the average non-reactive lepromatous patient small numbers of bacilli may constantly reach the surface of the skin.

In skin sections, bacilli are not infrequently found in the epidermis. Occasionally they have also been seen in the subcorneal layer and even in the corneal layer of the epidermis. Bacilli have also been found in the lumen of the sweat ducts and in the orifices of sweat ducts.

Though, admittedly, the number of bacilli thus reaching the surface of the skin is low as compared with the surface of nasal mucosa, the number is sufficiently 
high for the transmission of $M$. leprae. In experiments with mice it has been shown that multiplication of $M$. leprae can be obtained after inoculation of very small numbers of bacilli only (Shepard and McRae, 1965). Therefore, the transmission of $M$. leprae via the skin remains a definite possibility.

In the following section evidence is presented against transmission via the respiratory or gastro-intestinal tract.

\section{Clinical Course of Leprosy}

In a very high proportion of patients, probably in the great majority, the first clinical manifestation of the disease is a single skin lesion or, less frequently, a few skin lesions. In epidemics of leprosy and in children the first and only symptom of the disease is a single tuberculoid patch in a high proportion of the patients.

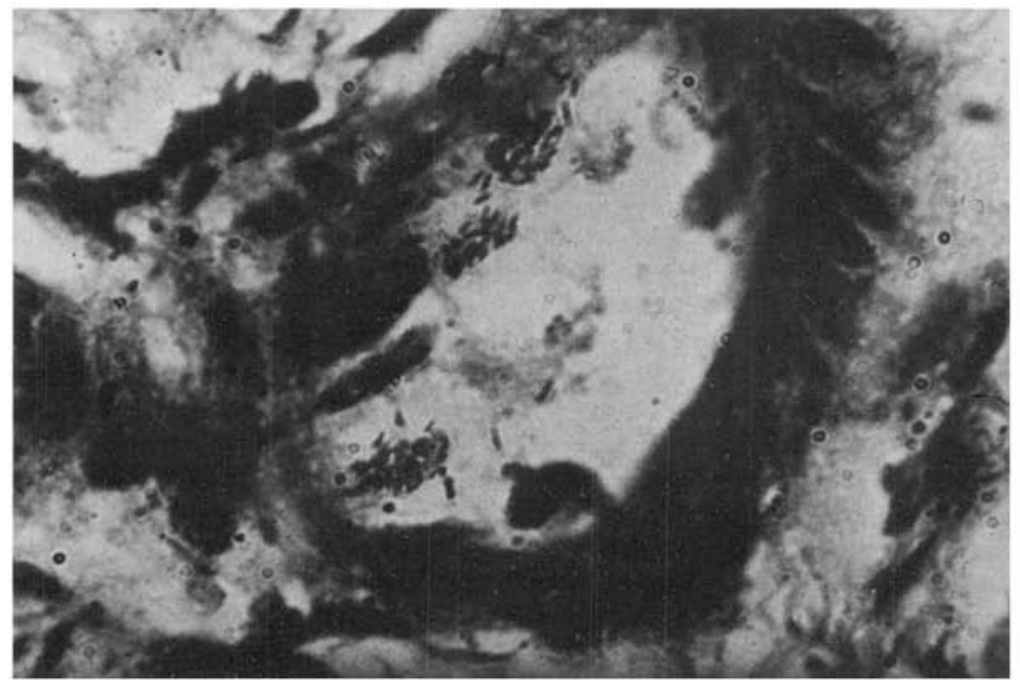

Fig. 1. Histopathological section showing M. leprae being released into a blood vessel. FFW. $\times 100$.

In a small proportion of the patients the disease is progressive. However, the single patch is usually not followed by a second single lesion, but by a crop of multiple new lesions, or by successive crops of new lesions. Such a course is compatible with the hypothesis that the first skin lesion is a primary lesion, indicating the site of inoculation, and with the new lesions being the result of haematogenous spread of several bacilli at a time from the primary lesion.

Histopathologically bacilli are frequently found in endothelial cells of blood vessels. It is likely that they are usually released only after the number has grown to the extent that the cell has been damaged, or after aging of the cell. Occasionally the release can be seen in histopathological sections (Fig. 1). Apparently it is the rule, rather than the exception that not one bacillus, but several bacilli are released at a time, producing multiple new lesions at different 
sites. The course of the disease is difficult to explain by the hypothesis that the primary lesion is located in the respiratory tract or in the gastro-intestinal tract and that the first skin lesions are in fact secondary lesions. It is inconceivable that so frequently only a single bacillus would escape from a lesion in these tracts, causing a single skin lesion, whereas so frequently many bacilli escape at a time from a first skin lesion causing multiple new skin lesions. The course of the disease is compatible with the hypothesis that the first skin lesion, as a rule, is a primary lesion.

\section{The Sites of the Skin Lesions}

The site of primary lesions is governed by the chance of inoculation. The site of secondary lesions, resulting from haematogenous spread, is influenced by the preference of $M$. leprae for the peripheral parts of the body. Consequently, a different distribution between the primary and secondary lesions in the individual patient, and a regional variation in the distribution of primary lesions is to be expected, the latter being influenced by various sociological factors such as customs influencing contact between people, the use of protective clothes, the mode of carrying and handling children, etc. The course of lower resistant (sub-polar) tuberculoid leprosy (Leiker, 1964) and of borderline tuberculoid leprosy, is illustrative.

Sub-polar tuberculoid leprosy usually starts with a single, or less frequently, with a few small indeterminate or tuberculoid lesions.

This first lesion may be located anywhere on the body. There is no obvious preference for the periphery of the body. Lesions on the trunk are about as common as those on the extremities or on the head. There is considerable regional variation in distribution. Single lesions on nose, ear, elbow, knee, hand or foot are not very common. In a high proportion of the patients the first lesion is followed .by one or more successive crops of new lesions. The new lesions, however, are not distributed at random. A definite preference for the periphery of the body is seen. After a single crop of new lesions in lower resistant tuberculoid leprosy, some lesions may be seen on the trunk, more of ten on the buttocks than elsewhere on the trunk and the distribution of the lesions remains asymmetrical. However, many more lesions are seen on the extremities, often with a marked tendency towards symmetry. Lesions are common on the hands, feet, elbows, knees, ears and the central part of the face, indeed "the peripheries of the peripheries" are predilection sites.

This distribution strongly suggests that the first skin lesion is a primary lesion and that the new lesions are secondary lesions resulting from haematogenous spread of bacilli from the first skin lesion. If the first lesions were the result of dissemination from a primary lesion in the respiratory tract or the gastrointestinal tract one would expect no difference in distribution between the first skin lesion and successive skin lesions and that both would show the same preference for the most peripheral parts of the body.

\section{Epidemiology}

In the last 30 years, in the Netherlands more than 200 immigrant patients with "open" leprosy, many of them not on treatment immediately after immigration and some on sulphone treatment but resistant to sulphones, have moved freely in 
the community. In the crowded streets, while using public transport, during daily labour in factories, at school, etc. the conditions for the spreading of an airborne causative agent undoubtedly are favourable. If leprosy is an airborne disease, many thousands of people must have been exposed to infection. However, only once has leprosy been diagnosed in a Dutchman who has never been in an endemic country. Only very few new patients have been found outside the families of known patients. Lack of susceptibility cannot be the explanation, judging from the prevalence rates in the different ethnic groups of immigrants (Indo-Europeans 1\%, Ambonese 1.5\%, West Indians 5\% ). The degree of susceptibility to $M$. leprae of the Dutch population is not known but the fact that at least 80 Dutch people contracted leprosy in endemic countries indicates that their susceptibility is far from negligible.

In the light of these observations, it appears most unlikely that in general the modes of spread of tuberculosis and leprosy are similar.

The history and the epidemiology of leprosy in parts of West New Guinea (Irian Yaja) were studied by Leiker (1960, 1971). It was possible to reconstruct in detail the course of the disease since its introduction into the community and to define with reasonable accuracy when each patient developed the first symptoms of the disease, what his family relationships were and in which house he had lived. In the Wandamen Bay area the disease was obviously largely focalized in certain families and in certain houses.

No correlation was found between the prevalence in one house and the unrelated neighbouring houses, but a correlation was present between one house and other, more distant, related houses. Family relationship, which primarily governs the degree of contact between people, was obviously more important than the distance between homes. Frequently a high prevalence was found in one clan and a low prevalence in another clan sharing the same village.

Difference in susceptibility is not a likely explanation of these findings, because once leprosy was introduced into a hitherto unaffected family, it usually spread within the family.

The population of Wandamen Bay lives in a row of a dozen villages along the beach. The administrative centre, shops, market and medical centre are in the 2 most northern villages. The third in the row is the leprosarium. Year after year, many people, who were living in the south, have made weekly visits to the northern villages, frequently taking the short cut through the leprosarium for visiting relatives or for reasons of convenience. If leprosy is an airborne infection one would expect a much more even distribution of the disease in the southern villages. The epidemiological evidence is not in favour of an airborne route of transmission.

\section{Gastro-intestinal Infection}

It is also not likely that leprosy is frequently transmitted from lepromatous mothers to their infants via the breast milk. Because of the large numbers of bacilli present in the milk glands one would expect transmission of $M$. leprae to occur soon after birth, and consequently a high incidence of leprosy in children around the age of 4-5 years. In practice, however, the incidence of leprosy in children below school age is relatively low. There is also no evidence of a particularly high incidence of leprosy in common feeding groups, or evidence that food, water or beer sellers suffering from leprosy are a high risk for their customers. 
Although it is likely that $M$. leprae is frequently inhaled or digested, the environmental conditions in the respiratory and gastro-intestinal tract are probably not favourable for survival of the bacilli.

The foregoing arguments do not challenge the importance of the nasal mucosa as a very important, if not the most important source of $M$. leprae.

If hygiene is deficient the skin may easily become contaminated with nasal discharge or saliva, whether directly or indirectly, e.g. by flies feeding on nasal discharge (Geater, 1975), and the number of bacilli thus reaching the skin may be higher than those reaching the surface by other routes.

\section{Recapitulation}

The importance of the nasal mucosa and the milk ducts of lactating mothers as sources of $M$. leprae is not challenged, neither the likelihood that $M$. leprae found on the skin may frequently have originated from the nasal mucosa. It does not however necessarily follow that as a general rule the primary lesion of leprosy is located in the respiratory or gastro-intestinal tracts and that skin lesions are secondary. On the contrary there is considerable evidence against this hypothesis.

\section{EPIDEMIOLOGICAL EVIDENCE}

(a) The course of leprosy in a closely observed area of Irian Yaja, Indonesia, over a period of 30 years does not appear to be consistent with transmission by droplet infection.

(b) In The Netherlands more than 200 patients with "open" leprosy have moved freely in a crowded community during the past 30 years but only one definite autochthonous case of leprosy has been found. This does not suggest that droplet infection is an important mode of transmission of leprosy.

(c) The average age at onset of the disease in children of lepromatous mothers does not correspond with the average incubation period of the disease. If the disease was transmitted by the breast milk during the early months of life one would expect more cases to arise at around 4-5 years of age, whereas in practice its onset is usually later than this.

\section{CLINICAL EVIDENCE}

(a) In a very high proportion of patients with tuberculoid leprosy, in particular in children, the first and only symptom of the disease is a single skin patch. In sub-polar and borderline tuberculoid leprosy the single first lesion is followed usually not by a single second lesion but by a crop of new skin lesions indicative of haematogenous spread of bacilli. Apparently several bacilli are usually released at a time from the primary lesion, wherever this is located. Such a course is compatible with the hypothesis that the first skin lesion is a primary lesion, because if the primary lesion was located in the respiratory or gastro-intestinal tract one would expect more frequently an onset of the disease with multiple skin lesions.

(b) The distribution of single lesions varies from area to area, but the peripheral parts of the body are not sites of predilection (Bechelli et al., 1973; Ganapati, 1976). The distribution of secondary skin lesions however shows a definite preference for the extremities and head. If the primary lesion was located in the internal tracts, and skin lesions are secondary, one would expect to find the first and single lesions predominantly at the periphery of the body. 


\section{BACTERIOLOGICAL EVIDENCE}

It has been shown from experiments with mice that inocula containing as few as 10-100 solid bacilli produced "takes" consistently and that the minimum infectious dose of solid bacilli was 3.4 to 34 (Shepard and McRae, 1965). The number of bacilli reaching the surface of the skin from ulcerating or ruptured vesicular lesions in reactive patients or those with histoid leproma, in addition to those present as a result of minor injury or contamination with nasal discharge or saliva, is sufficiently high to provide an adequate inoculum. Transmission via the skin therefore remains a definite possibility.

\section{The Need for Caution}

So far there is no conclusive evidence that leprosy is transmitted by droplet infection from one human being to another. Until such evidence exists the utmost discretion is needed in publicizing this possibility.

The present trends towards abandoning segregation and other restrictive practices on the part of health authorities have been hardly won, but have been of immense importance in the fight against leprosy. They must not be jeopardized except for the most compelling reasons. The suggestion that leprosy can be spread by droplet infection will have far-reaching effects. Implicit in it is the threat that restrictive measures should be re-introduced. This must profoundly affect the existing basis for leprosy control programmes which relies on the co-operation of patients in the early stages of their infection. With fear once again an important factor in their lives the motivation for co-operation disappears. The effect on the general public is equally profound. The admission of children with early leprosy to general schools, the employment of leprosy patients, the admission of leprosy patients to public places and public transport, even the willingness of nurses to care for leprosy patients in general hospitals; these are all facets of the situation which have to be reckoned with. Until such time, therefore, that conclusive evidence is found that leprosy can be spread by droplet infection from one person to another and the conditions in which this can occur are determined, great caution is needed in publicizing this hypothesis.

\section{References}

Bechelli, L. M., Gallego Garbajosa, P., Gyi Mg Mg., Martinez Dominguez, V. and Quagliato, R. (1973). Site of early skin lesions in children with leprosy. Bull. W.H.O. 48, 107.

Beek, C. H. (1961). An autochthonous case of leprosy in the Netherlands. Ned. Tÿdschrift. Geneeskd. 105, 2214.

Davey, T. F. (1957). Decline of leprosy in a group of Nigerian villages between 1941 and 1957. Int. J. Lepr. 25, 329.

Ganapati, R., Naik, S. S. and Pandya, S. S. (1976). Leprosy among school children in Greater Bombay: Clinical features. Lepr. Rev. 47, 133.

Geater, J. G. (1975). The fly as potential vector in the transmission of leprosy. Lepr. Rev. 46, 279.

Godal, T., Myrvang, B. and co-workers (1974). Recent advances in the immunology of leprosy with special reference to new approaches in immune-prophylaxis. Bull. de l'Inst. Pasteur $72,273$.

Leiker, D. L. (1960). Epidemiological and immunological surveys in Netherlands New Guinea. Lepr. Rev. 31, 241.

Leiker, D. L. (1964). Low resistant tuberculoid leprosy. Int. J. Lepr. 32, 359.

Leiker, D. L. (1971). Some aspects of the epidemiology of leprosy. Int. J. Lepr. 39, 610. 
Pedley, J. C. (1968). The presence of M. leprae in the breast secretion of a non-lactating woman with lepromatous leprosy. Lepr. Rev. 39, 111 .

Pedley, J. C. (1970). Summary of the results of a search of the skin surface for Mycobacterium leprae. Lepr. Rev. 41, 167.

Rees, R. J. W. and Meade, T. W. (1974). Comparison of the modes of spread and the incidence of tuberculosis and leprosy. Lancet 12, 47.

Shepard, C. C. and McRae, D. H. (1965). M. leprae in mice: Minimum infectious dose, relationship between staining quality and infectivity, and effect of cortisone. J. Bact. 89, 365.

Wade, H. W. and Ledowsky, V. (1952). The leprosy epidemic at Nauru; a review with data on the status since 1937. Int. J. Lepr. 20, 1. 